\title{
Взанмосвязь маркеров эндотелиальной дисфункции при гнойном холангите у больных с хроническим гепатитом и без хронического гепатита на фоне обтурационной желтухи
}

\author{
А. А. Мамедов, Э. А. Алиева, Н. М. Гасанов, Ф. С. Идрисов, Э. А. Искендеров \\ Научный центр хирургии имени М. А.Топчибашева, г. Баку, Азербайджанская Республика \\ Interrelationship of the endothelial dysfunction markers \\ in endothelial dysfunction in purulent cholangitis in patients, \\ suffering chronic hepatitis and without chronic hepatitis \\ on background of obturation jaundice
}

\author{
A. A. Mammedov, E. A. Alieva, N. M. Hasanov, F. S. Idrisov, E. A. Iskenderov \\ Topchibashev Scientific Centre of Surgery, Baku, Azerbaijani Republic
}

\section{Реферат}

Цель. Изучение взаимосвязи маркеров эндотелиальной дисфункции при гнойном холангите у больных с хроническим гепатитом и без хронического гепатита на фоне обтурационной желтухи, выявление его раннего перехода в цирроз печени и оптимизация тактики лечения в до- и послеоперационном периоде с оценкой показателей исследуемых лабораторных тестов.

Материалы и методы. Исследование проведено в отношении 15 больных с гнойным холангитом, из них у 9 больных был хронический гепатит, у 6 больных хронического гепатита не было. Из 9 больных с хроническим гепатитом 4 больным противовирусную терапию не проводили, 5 - проводили.

Результаты. Непосредственные результаты проведенного лечебно-диагностического исследования свидетельствуют о сходных проявлениях эндотелиальной дисфункции при гнойном холангите у больных с хроническим гепатитом и без хронического гепатита.

Всем больным с гнойным холангитом выполняли декомпрессию желчных протоков, устраняли причины обтурации, проводили дезинтоксикационную терапию.

По данным корреляционного анализа показателей функционального состояния эндотелия у больных с хроническим гепатитом, получавших противовирусную терапию, улучшение отметили на 10-е сут, через 1 мес оно было более выраженным, чем у больных, не получавших противовирусную терапию.

У 6 больных с гнойным холангитом без хронического гепатита своевременное хирургическое, дезинтоксикационное лечение совместно с озонотерапией привело к улучшению показателей функционального состояния эндотелия на 10-е сут, через 1 мес они приблизились к норме.

Выводы. Своевременное выявление эндотелиальной дисфункции при гнойном холангите у больных с хроническим гепатитом и без хронического гепатита способствует раннему прогнозированию его перехода в стадию цирроза печени и правильному выбору тактики лечения в до- и послеоперационном периоде. Подытожив полученные данные, можно сделать вывод о том, что при гнойном холангите у больных с хроническим гепатитом целесообразно проведение противовирусной терапии до и после операции.

ключевые слова: маркеры эндотелиальной дисфункции; гнойный холангит; противовирусная терапия.

Abstract

Objective. Studying of interrelationship of the endothelial dysfunction markers in endothelial dysfunction in purulent cholangitis in patients, suffering chronic hepatitis and without chronic hepatitis on background of obturation jaundice, revealing of its early transition into hepatic cirrhosis and optimization of the treatment tactics in pre- and postoperative periods with estimation of the laboratory tests indices investigated.

Materials and methods. The investigation was conducted in 15 patients with purulent cholangitis, of them in 9 patients was chronic hepatitis, in 6 patients chronic hepatitis was absent. Of 9 patients, suffering chronic hepatitis, in 4 antiviral therapy was not conducted, while in 5 - conducted.

Results. Immediate results of the diagnosis-treatment investigation conducted witnesses about similar signs of endothelial dysfunction in purulent cholangitis in patients, suffering chronic hepatitis and without chronic hepatitis.

In all the patients, suffering purulent cholangitis, decompression of biliary ducts was accomplished, the obturation causes were eliminated, detoxication therapy was conducted.

In accordance to correlation analysis of indices of the endothelium functional state in patients, suffering chronic hepatitis, who obtained antiviral therapy, improvement was noted on the $10^{\text {th }}$ day, in $1 \mathrm{mo}$ it was more significant, than in patients, who did not obtained antiviral therapy.

In 6 patients, suffering purulent cholangitis without chronic hepatitis, a timely surgical, desintoxication treatment with ozonotherapy have resulted in improvement of the endothelial functional state indices on the $10^{\text {th }}$ day, in 1 mo they have approached to norm. Conclusion. A timely revealing of endothelial dysfunction in purulent cholangitis in patients, suffering chronic hepatitis and without chronic hepatitis, promotes an early prognostication of its transition into stage of hepatic cirrhosis and correct selection of the treatment tactics before and after the operation. Here may be concluded, that in purulent cholangitis in patients, suffering chronic hepatitis, it is expedient to conduct antiviral therapy before and after the operation.

Keywords: markers of endothelial dysfunction; purulent cholangitis; antiviral therapy. 
В последние 10 лет доказана патогенетическая роль эндотелиальной дисфункции при ряде наиболее распространенных заболеваний, патологических состояний, в том числе при хроническом гепатите (ХГ) и циррозе печени, но фактически нет сведений о взаимосвязи маркеров эндотелиальной дисфункции при гнойном холангите (ГХ) у больных с ХГ и без ХГ на фоне обтурационной желтухи.

Безусловно, в патогенезе ГХ и прогрессировании его с переходом в хроническую патологию печени (цирроз печени) большое значение имеет эндотоксиновая агрессия, обусловленная холангиогенной инфекцией и оксидантным стрессом, гипербилирубинемией, а в патогенезе вирусного ХГ и прогрессировании его с переходом в цирроз печени - нарушение внутрипеченочной гемодинамики, что может быть связано с повреждением эндотелиальной выстилки печеночных синусоидов непосредственно вирусом и развитием эндотелиальной дисфункции $[1,2]$. Под воздействием вируса на эндотелий синусоидов печени способность эндотелиальных клеток освобождать релаксирующие факторы уменышается, тогда как образование сосудосуживающих факторов увеличивается, снижается барьерная функция эндотелия и повышается его проницаемость для провоспалительных цитокинов, нарушается биодоступность оксида азота (NO), являющаяся одним из важных факторов противовирусной защиты [2]. При тяжелом поражении эндотелия нарушается его целостность и в интиме появляются участки, лишенные эндотелиальной выстилки. Процессы прогрессирования поражения печени, в частности неоангиогенез и фиброз, в свою очередь связаны с функциональной несостоятельностью эндотелия. Таким образом, эндотелий играет ключевую роль в патогенезе вирусных поражений печени $[1,3,4]$.

Известно, что эндотелиальная дисфункция - это предиктор и неизменный компонент патогенеза многих заболеваний. Под этим термином понимают дисбаланс между веществами, вырабатываемыми эндотелием. При нормальной функции эндотелия баланс всегда сдвинут в сторону поддержания вазодилатации, ингибиции ангиогенеза, антитромботического, противовоспалительного, антиоксидантного эффекта. Наиболее важные маркеры функционального состояния эндотелия - NO, эндотелин-1 (Et-1), десквалимированные эндотелиоциты, фактор Виллебранда (ФВ) $[4,5]$.

Синтез NO при дисфункции эндотелия нарушается, что ведет к снижению его концентрации в плазме крови. Нормальная функция NO заключается в атромбогенности сосудистой стенки, где наблюдается ее нарушение. Этот фактор также называют фактором релаксации [3, 6].

ФВ накапливается в эндотелии и выделяется из него при стимуляции или повреждении. Нормальная функция ФВ - выполнять роль «реологического клея», соединяя рецепторы тромбоцитарной мембраны с субэндотелиальными структурами поврежденной стенки сосуда. Отдельными исследованиями установлено закономерное нарастание уровня ФВ в плазме крови при значительном сосудистом поражении [2,3].

Et-1 в норме практически не синтезируется, но его содержание резко увеличивается при стимуляции эндоте- лия $[2,3,7,8]$. Et-1 является антагонистом NO, который влияет на тонус сосудов [4]. Это мощный вазоконстрикторный пептид, который, помимо этого, регулирует ангиогенез сосудов. Et-1 образуется под действием многих факторов (адреналина, тромбина, ангиотензина, вазопрессина) и секретизируется внутри сосудистой стенки, где расположены специфические высокоаффинные рецепторы. В ряде работ отмечено повышение концентрации Et-1 при некоторых инфекционных заболеваниях [4,7]. Можно сделать вывод, что Et-1 играет определенную роль в модуляции сосудистой резистентности печени, хотя значение его в патогенезе некоторых гнойных заболеваний, например ГХ, пока не совсем ясно.

Значение перечисленных факторов в развитии дисфункции эндотелия при ГХ у больных с ХГ и без ХГ, раннее прогнозирование исхода с повреждением печени и оптимизация правильного выбора тактики лечения нуждаются в дальнейшем изучении.

Цель исследования: изучение взаимосвязи маркеров эндотелиальной дисфункции при ГХ у больных с ХГ и без ХГ на фоне обтурационной желтухи, выявление его раннего перехода в цирроз печени и оптимизация тактики лечения в до- и послеоперационном периоде с оценкой результатов исследуемых лабораторных тестов.

\section{Материалы и методы исследования}

Для достижения цели исследования 15 больных разделили на две группы. Основную группу составили 9 больных с острым ГХ и ХГ. Данную группу разделили на две подгруппы: 1-я подгруппа - 4 больных, которым в преди послеоперационном периоде не проводили противовирусную терапию (ПВТ); 2-я подгруппа - 5 больных, которым ПВТ проводили. Контрольную группу составили 6 больных с ГХ без ХГ. Всем 15 больным проведено двухэтапное хирургическое лечение.

Помимо общеклинического (общий и биохимический анализ крови, коагулограмма) и инструментального (ультразвуковое исследование, сцинтиграфия) обследования и выявления серологических маркеров вирусных гепатитов ( $\mathrm{HB}_{\mathrm{s}} \mathrm{A}_{\mathrm{g}}$ и анти - HCVg $\left.\operatorname{IgG} / \mathrm{IgM}\right)$, мы изучали с помощью метода иммуноферментного анализа функциональное состояние эндотелия, определяя в сыворотке крови уровни общего NO (Systems, CША), Et-1(BiomedicaGruppe, США), функциональную активность ФВ (Technoklon;WR; CBA; ELICA; СШA).

Результаты обрабатывали при помощи пакета программ Statistica 6,0. Для статистической обработки данных использовали метод вариационной статистики с применением t-критерия Стьюдента.

\section{Результаты}

На основании изучения лабораторных тестов функционального состояния эндотелия при ГХ с ХГ и без ХГ были выявлены лабораторные признаки поражения эндотелия (табл. 1). При ГХ у больных с ХГ уровень NO снижался более выраженно, чем у больных без ХГ ( $<$ < 0,001). Уровень Et-1 у больных с ХГ повышался более значительно, чем у больных без ХГ ( $\mathrm{p}<0,007)$, что указывает на дисбаланс вазоактивных соединений и нарушение кровоо- 
бращения в печени. Эти изменения у больных с ГХ и ХГ были более выражены. В обеих группах функциональная активность ФВ, который относится к маркерам повреждения эндотелия, повышалась, однако у больных с ГХ и ХГ больше по сравнению с нормой, чем у больных без ХГ ( $<$ 0,002), и являлась признаком слущивания эндотелиоцитов, неоангиогенеза, активации адгезии и агрегации тромбоцитов. Между двумя группами обнаружены значимые различия, однако степень повреждения эндотелия, несомненно, играет важную роль в патогенезе указанных заболеваний.

Таким образом, поражение эндотелия закономерно нарастает при прогрессировании ГХ у больных с ХГ, усиливается активность вируса и возможность формирования цирроза печени больше, чем у больных без ХГ.

По данным функционального клинического исследования коэффициент де Ритиса (соотношение активности сывороточных аспартатаминотрансферазы и аланинаминотрансферезы), показатели коагулограммы, свертываемости крови при ГХ в обеих группах фактически не отличались, несмотря на более выраженную эндотелиальную дисфункцию у больных с ГХ и ХГ.

Анализ показателей маркеров дисфункции эндотелия при ГХ в обеих группах позволил нам выбрать правильную тактику лечения.

Кроме двухэтапного оперативного лечения с применением современных технологий и стандартных хирургических вмешательств для детоксикации, улучшения оттока желчи, уменышения интоксикации с применением дезинтоксикационной терапии, мы одновременно проводили комбинированную ПВТ препаратами интерферона в сочетании с рибавирином в до- и послеоперационном периоде.

При ГХ у больных с ХГ, которым проводили комбинированную ПВТ (табл. 2), уровень NO достоверно повы- сился на 10-е сут на 70\%, через 1 мес - на 89\%; концентрация Et-1 уменьшилась на 10-е сутки на 80\%, через 1 мес - на 90\%; функциональная активность ФВ уменьшилась на 10-е сут на 30\%, через 1 мес - на 75\%. У больных, которым не проводили ПВТ, на 10-е сут уровень NO повысился на 57\%, через 1 мес - на 69\%; концентрация Et1 уменьшилась на 10-е сут на 60\%, через 1 мес - на 70\%; функциональная активность ФВ уменьшилась на 10-е сут на 25\%, а через 1 мес - на 55\%. Это свидетельствует об уменьшение повреждения и дисфункции эндотелия в обеих подгруппах, но оно было более выраженным во 2-й подгруппе, у этих больных все показатели почти приблизились к норме.

При ГХ у больных без ХГ, которым проводили двухэтапное хирургическое лечение для детоксикации и улучшения оттока желчи, уменьшения интоксикации с применением дезинтоксикационной терапии, на 10-е сут уровень NO достоверно повысился на 80\%, через 1 мес - на 99,9\% (почти до нормы), концентрация Et-1 уменьшилась на 10-е сут на 90\%, через 1 мес она нормализовалась, функциональная активность ФВ уменьшилось на 10-е сут на 60\%, а через 1 мес - на 100\%.

\section{Обсуждение}

Результаты исследования свидетельствуют о сходных проявлениях поражения эндотелия при ГХ у больных с ХГ и без ХГ. Эти заболевания протекают с первичным либо вторичным поражением паренхимы печени на фоне воспалительной активности и функциональных нарушений гепатоцитов, что приводит к развитию эндотелиальной дисфункции и ухудшению кровообращения в печени.

Безусловно, выраженность эндотелиальных нарушений адекватно отражается на тяжести поражения печени [4]. Полученные результаты исследования свидетель-

\begin{tabular}{|c|c|c|c|}
\hline \multirow{2}{*}{ Показатели } & \multirow{2}{*}{ Норма } & $\Gamma X(n=15)$ & \\
\hline & & $c X \Gamma(n=9)$ & без ХГ $(n=6)$ \\
\hline NO, мкмоль/л & $29,43 \pm 3,34$ & $11,07 \pm 4,42$ & $18,7 \pm 2,02$ \\
\hline Et-1 фмоль/л & $0,3 \pm 0,13$ & $1,6 \pm 0,52$ & $0,62 \pm 0,20$ \\
\hline$\Phi \mathrm{B}, \%$ & $55,5 \pm 37,9$ & $185,4 \pm 25,2$ & $139,8 \pm 60,6$ \\
\hline
\end{tabular}

\begin{tabular}{|c|c|c|c|c|c|c|c|c|c|}
\hline \multirow{5}{*}{ Показатели } & \multirow{5}{*}{ Норма } & \multicolumn{8}{|c|}{$\Gamma X$} \\
\hline & & \multicolumn{5}{|c|}{ c XГ } & \multicolumn{3}{|c|}{ без ХГ } \\
\hline & & \multirow{3}{*}{$\begin{array}{c}\text { до лечения } \\
\quad(\mathrm{n}=9)\end{array}$} & \multicolumn{4}{|c|}{ после лечения } & \multirow{3}{*}{$\begin{array}{c}\text { до лечения } \\
(\mathrm{n}=6)\end{array}$} & \multirow{2}{*}{\multicolumn{2}{|c|}{$\begin{array}{c}\text { после лечения } \\
(n=6)\end{array}$}} \\
\hline & & & \multicolumn{2}{|c|}{$\begin{array}{c}\text { 1-я подгруппа } \\
(n=4)\end{array}$} & \multicolumn{2}{|c|}{$\begin{array}{c}\text { 2-я подгруппа } \\
(n=5)\end{array}$} & & & \\
\hline & & & 10-е сут & $\begin{array}{l}\text { через } \\
1 \text { мес }\end{array}$ & 10-е сут & $\begin{array}{l}\text { через } \\
1 \text { мес }\end{array}$ & & 10-е сут & $\begin{array}{l}\text { через } \\
1 \mathrm{мес}\end{array}$ \\
\hline \multirow{2}{*}{$\begin{array}{c}\mathrm{NO}, \\
\text { мкмоль/л }\end{array}$} & \multirow[t]{2}{*}{$29,43 \pm 3,3$} & $11,07 \pm 4,4$ & $16,6 \pm 2,3$ & $21,7 \pm 3,3$ & $19,82 \pm 2,7$ & $25,5 \pm 4,6$ & $18,7 \pm 2,0$ & $20,9 \pm 2,1$ & $28,9 \pm 1,6$ \\
\hline & & $p<0,001$ & $p<0,005$ & $p<0,001$ & $p<0,005$ & $p<0,001$ & $p<0,001$ & $p<0,001$ & $p<0,001$ \\
\hline \multirow{2}{*}{$\begin{array}{c}\text { Et-1, } \\
\text { фмоль/л }\end{array}$} & \multirow[t]{2}{*}{$0,3 \pm 0,1$} & $1,6 \pm 0,5$ & $0,9 \pm 0,2$ & $0,7 \pm 0,5$ & $0,8 \pm 0,25$ & $0,5 \pm 0,01$ & $0,62 \pm 0,2$ & $0,5 \pm 0,5$ & $0,3 \pm 0,1$ \\
\hline & & $p<0,007$ & $p<0,001$ & $p<0,001$ & $p<0,001$ & $p<0,001$ & $p<0,007$ & $p<0,001$ & $p<0,001$ \\
\hline \multirow[t]{2}{*}{$\Phi \mathrm{B}, \%$} & \multirow[t]{2}{*}{$55,5 \pm 37,9$} & $185,4 \pm 3,2$ & $170,2 \pm 22,3$ & $132,0 \pm 1,6$ & $150,9 \pm 1,1$ & $105 \pm 0,4$ & $139,8 \pm 60,6$ & $92,5 \pm 1,5$ & $56,2 \pm 1,3$ \\
\hline & & $p<0,001$ & $p<0,001$ & $p<0,001$ & $p<0,001$ & $p<0,001$ & $p<0,002$ & $p<0,001$ & $p<0,001$ \\
\hline
\end{tabular}


ствуют о том, что дисфункция эндотелия при ГХ развивается вследствие прогрессирования эндотоксиновой агрессии, обусловленной холангиогенной инфекцией и оксидантным стрессом. Но при прогрессировании ГХ у больных с гепатитом усиливается активация вируса, которая непосредственно приводит к повреждению эндотелиальной выстилки печеночных синусоидов, что соответствует данным, полученным А. П. Щекотовой и соавторами [7]. У больных с ХГ, получавших ПВТ в до- и послеоперационном периоде, по данным корреляционного анализа показателей функционального состояния эндотелия улучшение было более выраженным, чем у больных, не получавших ПВТ.

При ГХ без ХГ своевременное хирургическое лечение и дезинтоксикационная терапия совместно с озонотерапией улучшают показатели функционального состояния эндотелия, которые приближаются к норме.

\section{Выводы}

1. Полученные данные дают основание рекомендовать при ГХ у больных с ХГ проводить в обязательном порядке ПВТ до и после операции.

2. Своевременное выявление дисфункции эндотелия при ГХ и правильный выбор лечения в до - и послеоперационном периоде предотвращают прогрессирование ГХ до развития хронической патологии печени (цирроза печени).

3. Перспективы дальнейших исследований заключаются в том, что, помимо общеклинического и инструментального обследования при ГХ у больных с ХГ и без ХГ, необходимы выявление маркеров вирусных гепатитов в сыворотке крови и оценка функционального состояния эндотелия, что позволит своевременно выбрать правильную тактику лечения и предотвратить поражение печени.

\section{References}

1. Shhekotova AP. Disfunkcija i povrezhdenie jendotelija pri gepatobiliarnoj patologii. Ural'skij medicinskij zhurnal. 2010;(1):96-9. [In Russian].

2. Friedrich-Rust M, Rosenberg W, Parkes J, Herrmann E, Zeuzem S, Sarrazin C. Comparison of ELF, Fibro Test and Fibro -Scan for the noninvasive assessment of liver fibrosis. BMC Gastroenterology. 2010;(10):103. doi: org/10.1186/1471-230X-10-103.

3. Zvjagincev TD, Gridneva SV. Sovremennye predstavlenija o sosudistom jendotelija v norme i pri patologii zheludochno-kishechnogo trakta. Jeksp Klin Gastrojenterol. 2005;(4):6-12. [In Russian].

4. Tomas H, Baldus S, Rudolph V, Yskert von Kodolitsch, Volker R, Thomas M. Systemic endothelial dysfunction as an early predictor of adverse outcome in heart failure. Article in Arteriosclerosis Thrombosis and Vascular Biology. 2005 June;25(6):1174-9. with 102 Reads. doi: 10.1161/01. ATV.0000166516.52477.81.

5. Rodford JL, Torrens C, Siow RC, Mann GE, Hanson MA, Clough GF. Endothelial dysfunction and reduced antioxidant protection in an animal model of the developmental origins of cardiovascular disease. J Physiol. 2008 Oct 1;586(19):4709-20. doi: 10.1113/jphysiol.2008.156976.

6. De Castro IF, Micheloud D, Berenguer J, Guzmán-Fulgencio M, Catalán $\mathrm{P}$, Miralles $\mathrm{P}$, et al. Hepatitis $\mathrm{C}$ virus infection is associated with endothelial dysfunction in HIV/hepatitis $\mathrm{C}$ virus coinfected patients. CLINICAL SCIENCE. AIDS. 2010 Aug 24;24(13):2059-67. doi: 10.1097/ QAD.0b013e32833ce54d.

7. Shhekotova AP, Kotel'nikova LP, Mugatarov IN, Fedachuk NN. Jendotelial'naja disfunkcija vospalenie i fibroz pri gepatobiliarnoj patologii. Fundamental'nye issledovanija. 2013;5(2):451-5. [In Russian].

8. Non WM, Lee WH, Khoo HE. Nitric oxide in liver diseases:friend, foe, orjust, passerby. New York Academy of Sciences. 2002;962:275-95. doi: org/10.1111/j.1749-6632.2002.tb04074.x. 